\title{
High-Resolution Synchronous Digital Ballistocardiography Setup
}

\author{
Nico Jähne-Raden ${ }^{\mathrm{a}}$, Ulf Kulau ${ }^{\mathrm{b}}$, Henrike Gütschleg ${ }^{\mathrm{a}}$, \\ Thiemo Clausen ${ }^{\mathrm{b}}$, Tobias Jura ${ }^{\mathrm{b}}$, Stephan Sigg ${ }^{\mathrm{c}}$, Lars Wolf ${ }^{\mathrm{d}}$ \\ ${ }^{a}$ Peter L. Reichertz Institute for Medical Informatics, Germany \\ ${ }^{b}$ Institute of Computer Engineering, TU Braunschweig, Germany \\ ${ }^{\mathbf{c}}$ Department of Communications and Networking, Aalto University, Finland \\ d Institute of Operating Systems and Computer Networks, TU Braunschweig, Germany
}

\begin{abstract}
Ballistocardiography (BCG) describes the measurement and analysis of ballistic forces that are evoked by the heart's movement and propagated to the surface of the body. Although there are several research groups focusing on BCG, the understanding on general BCG signals is still limited, as existing studies only consider a small number of subjects and insufficient measurement setups.

This paper presents first results of our ongoing study to collect BCG ground-truth data. We present a custom hardware implementation for both, high data quality and simultaneous ECG reference measurement. In a large-scale study we currently collected more than 10 hours of BCG data from 34 subjects including BCG in resting position as well as under physical stress. In addition, related biometric data are collected to enable comprehensive investigations. We plan to provide access to the data for interested researchers.
\end{abstract}

\section{Introduction}

Hardware-software systems, e.g., including accelerometers, are a vital part of medical diagnostics. However, the use of accelerometers is usually reduced to rather simple aggregation of movement data, for example activity determination. But with the advancing technical developments, digital accelerometers become both, highly integrated and affordable. Thus, nowadays accelerometers a usable for further medical diagnostics, e.g., for Ballistocardiography (BCG).

$\mathrm{BCG}$ is a method to gain detailed information about body movements imparted by the ballistic forces associated with cardiac contraction, the ejection of blood and with the deceleration of blood flow through the large blood vessels. These heart-related movements can be measured by Micro-ElectroMechanical-Systems (MEMS), such as digital accelerometers. The mass of such sensor devices has become insignificant so that a measurement of the proper acceleration is possible on the body's surface even for non-zero-g environments (see [1]).

A measurement on the chest surface is called Seismocardiography (SCG) while BCG includes also areas of the body which are more distant from the heart, like limbs or even proximal structures like chairs or beds [2]. However, the acceleration is rather small and highly sensitive sensor devices are required to measure tiny amplitudes with a low noise ratio. In addition, low costs are desired to enable a wide use of BCG for medical diagnostics in future systems. Thus, we deal with inexpensive, digital and commercial-of-the-shelf Computing in Cardiology 2019; Vol 46
(COTS) 3D-accelerometers to measure the momentum generated by the heartbeat. By using multiple accelerometers at different positions on a body, the time difference between signals (pulse transit time) can further be used to estimate the blood pressure $(n=15$, analog sensors, each subject one time for $60 \mathrm{~s}, \mathrm{ECG}+\mathrm{BCG}+$ blood pressure cuff, $1 \mathrm{kHz}$ ) (see [3]). A non-invasive measurement of blood pressure related parameter which is much less disturbing than the conventional methods and can therefore be used for long-term blood pressure monitoring even while sleeping.

From a system perspective more, requirements have to be considered, e.g., size, weight, energy-efficiency and communication capabilities. Depending on the specific choices, various application scenarios become possible. For instance, by integration into clothes, i.e., with wearables containing BCG systems, the health condition of people could be monitored in everyday life, for example to detect serious heart diseases at early stage. Further communication to medical specialists could support diagnostics remotely, thus extending telemedicine.

We use an FPGA board that is able to read-out several digital sensors (accelerometers and ECG) synchronously with high sample rates and resolutions. An ongoing largescale study with currently more than 20 subjects is performed to enable a deep investigation of BCG based on a best possible dataset.

Within the described project we build up a first foundation of multiple position BCG measurement database with suitable reference signals at a sample rate, so high chosen, that the entropy of the BCG signal can analyzed in the future and the optimal sample frequency can be evaluated. With this data the ground truth of a "healthy" BCG signal over a bigger group of subjects (more than 20) with current digital accelerometer technology can be realized.

\subsection{Related Work}

The investigation of BCG for medicine and the connection of BCG to heart function is a current research topic. Di Rienzo et al. developed a combined sensor system consisting of ballisto-/seismocardiographic sensors and an ECG. They recorded and analyzed the data of humans over 24 hours $(\mathrm{n}=5$, analog sensors, each subject 24h, 1-lead ECG + SCG, $200 \mathrm{~Hz}$ ) [4]. In addition, they studied the beat-to-beat BCG signal on ambulant subjects including sleep periods $(n=1$, analog sensors, 8h, 1-lead ECG + SCG, 200Hz). The results revealed the feasibility to estimate the relation between pulse transit time (PTT) and blood pressure $(n=1$, analog sensors, one-time 25min, ECG + SCG + PPG). Furthermore, first trails to estimate BCG signals while slow pace walking is Page $t_{i z e d} b$ ISSN 
4min for each subject, ECG + BCG + ICG, 2kHz) [5-8].

In our previous work [9] we already analyzed several digital accelerometers regarding their usability to measure BCG signals. A pre-selection based on the specifications provided in the datasheets, as well as an extensive laboratory test revealed the relevant parameters to select BCG capable digital accelerometers. For the determination of measurement position we mounted a group of overall nine BCG arrays on young, healthy subjects [9]. We further analyzed the possibility of signal differencing between similar signals in terms of morphology [10]. However, also this study lacks in terms of data quality and a limited number of subjects.

In order to make BCG signals available for medical care in the future, it is necessary to collect information about BCG data from a wide range of people. Starting with the efforts described here, we want to create an open database for BCG data of heart healthy people (recorded by assessments and checked physicians). With this database and future extensions, it should be possible to gain more information about these possible diagnostics by using established signal processing methods.

With more knowledge about the BCG and SCG signals and their relation to the actual heart movement, it should be possible to provide methods for heart diagnostics, as well as long-term health monitoring also in the everyday life, like public seats (e.g. on a plane) [11].

Considering the current state of research, BCG is still a young branch of research. So far, the general feasibility of BCG has been shown and existing work revealed possible correlations to other biometric data, e.g. ECG. Nevertheless, the test methodology of previous studies is not suitable to drain universal statements about BCG signals. From these previous studies, the data quality is not sufficient (e.g. all referenced articles did not describe of synchronicity of the different sensors), the test procedures are too vague (e.g. all referenced articles used no assessments), and the number of cases is too low. These are the major reasons why our work aims to build up a database with BCG ground-truth data to allow for future BCG investigations.

\section{Methods}

In this work we want to achieve a BCG measurement at its best. The gathered data should be at highest standard in terms of data quality, including high resolution, high sample rate and real-time guarantees. Thus, the goal is to provide a sustainable data base that will enable further research at the highest stage.

On first sight the measurement of BCG data and further vital parameters (i.e. ECG) sounds like a regular engineering task for computer scientists. The sensors are equipped with common interfaces like I2C, SPI or analogue outputs. When using multiple sensors, e.g. to enable the measurement at different locations of the body, the buses (I2C, SPI) of an MCU can be shared and analogue channels could be multiplexed. In sum, using regular MCUs will work but a synchronous signal recording cannot be guaranteed. Moreover, due to the sequential readout of the sensors only low data rates can be achieved.

However, we are not able to achieve this goal with standard hardware components. For this reason, we rely on an FPGA implementation for a synchronous data collection with precise time-stamping. In particular we are using a Xilinx Zynq-7020 system on chip (SoC) that combines a dual core ARM processor with a Virtex 7 FPGA. At current stage we rely on the Zedboard Evaluation Board (see Figure 1) as it already offers several useful peripherals, for example Ethernet.

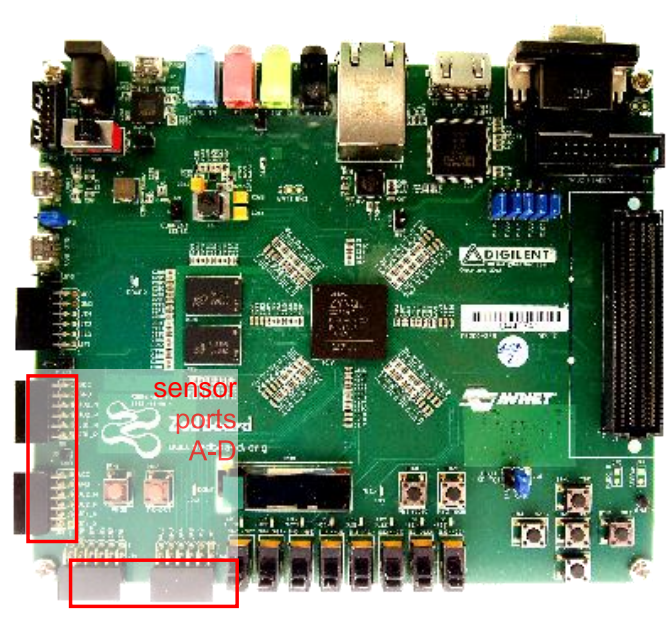

Figure 1: Zedboard with port markings

For interfacing the sensors, we implemented a hardware module on the FPGA that allows the utilization of parallel SPI controllers for each sensor. All SPI controllers use a common clock so that e.g. the readout of data is absolutely synchronous. As the parallel SPI controllers are implemented in hardware, a high sampling rate can be achieved (up to $20 \mathrm{kHz}$ ).

Each sample is additionally time-stamped in hardware and forwarded to a buffer and a DMA stream. The DMA stream is processed by the ARM cores and further forwarded to the processing/storing system.

The following Figure 2 illustrates a general block diagram of the SoC based measurement system. As can be seen in this diagram all four sensors (3x accelerometers and the 6-Channel-ECG) are read in parallel by using the SPI connection, while the HW timestamping is done for each channel (12 in total). By storing the digital sensor data in a buffer and providing the data to the system a transfer per TCP via ethernet is realized to feed a database server.

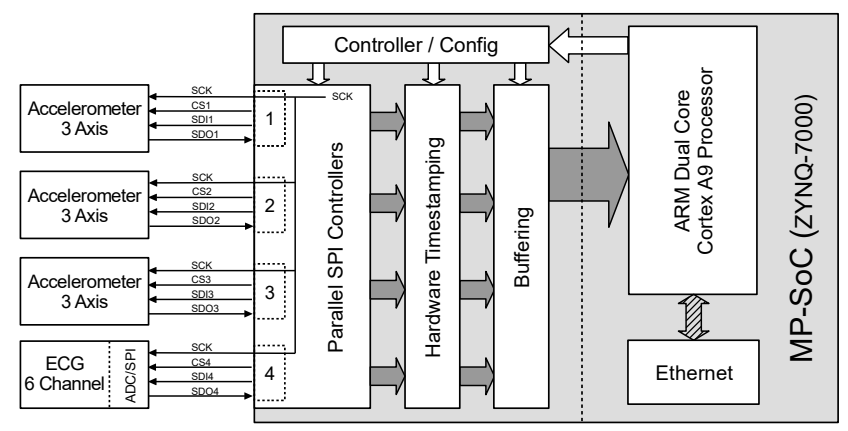

Figure 2: Diagram of the BCG system including the Zedboard and the sensors

The selection of acceleration sensors is based on an extensive technology review. Included were all digital, triaxial accelerometers found on established platforms (136 sensor entities of 13 manufacturers). Based on our previous study we know that the focus of the selection should be on the sensitivity [9]. For similar or equal sensitivity, next selection parameter, if available, was the noise density, alternatively the noise level. Finally, the choice was limited to five MEMS, since the scope of the pre-human measurement. For this final selection the availability as well as the price were considered. This selection attempts to present an up-to-date and realistic illustration of the acceleration sensors actually used in research and industry.

The five different acceleration sensors (see Table 1) are used on custom breakout boards to achieve a structural Pagequality in terms of size and weight (see Figure 3). 
Our system supports the following digital accelerometers:

\begin{tabular}{|c|c|c|c|}
\hline Sensor & $\mathbf{\pm g}$ & Sensitivity & N-D/Noise \\
\hline ADXL313WACPZ-RL7 & $\begin{array}{c}0.5,1, \\
2,4\end{array}$ & $115-1126 \mathrm{LSB} / \mathrm{g}$ & $150-250$ \\
\hline BMA456 & $\begin{array}{c}2,4,8, \\
16\end{array}$ & $2048-16384 \mathrm{LSB} / \mathrm{g}$ & 120 \\
\hline MMA9550LR1 & $2,4,8$ & $4098-16393 \mathrm{LSB} / \mathrm{g}$ & 100,120 \\
\hline KX122-1037 & $2,4,8$ & $16-6384$ counts/g & $0.75 \mathrm{mg} @ 50 \mathrm{~Hz}$ \\
\hline LIS2DS12TR & $\begin{array}{c}2,4,8, \\
16\end{array}$ & $2049-16393 \mathrm{LSB} / \mathrm{g}$ & $120-300$ \\
\hline
\end{tabular}

Table 1: Listing of the chosen BCG accelerometers for the measurements

An emerging limitation of this structural equality is the actual size or weight of the sensor itself. For example, the dimension of the Analog Devices Sensor is $5 \times 5 \times 1.45 \mathrm{~mm}$ while the Kionix sensor is only $2 \times 2 \times 0.9 \mathrm{~mm}$. In previous studies we were able to show that better BCG measurement results can be achieved with light sensors. However, these small differences are negligible in terms of low mass <30 mg.

Because of the similar measurement results in terms of signal to noise ratio, we chose the KX122 for the large-scale study. The breakout boards are mounted by using medical tape.

For the BCG measurements the highest possible solution shall be used (should be at least 14 up to 16 bit) and a sample frequency of at least $500 \mathrm{~Hz}$ (most accelerometers offer 800 $\mathrm{Hz}$ up to $1 \mathrm{kHz}$ ) was mentioned by cardiologists, yet never justified.

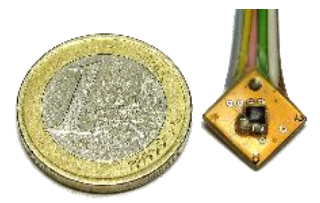

Figure 3: KX122 breakout boards with coin for scale

As an ECG reference, we have selected a simple ECG shield with 3 electrodes for the derivation of the electrical cardiac signals according to Einthoven and Goldberger. This 6-ch. ECG is an appropriate method to obtain signal references to the actual muscle contraction that will be measured across the trials.

The OLIMEX-Shield offers an analog output, which is converted into a digital signal by a SPI-ADC. Thus, the ECG signal can be recorded simultaneously to the accelerometers. The data resolution of the ECG board is at 10bit for each channel with a maximum sample frequency of the Zedboard itself, caused by the used analogue output using a MCP3008 ADC.

For each subject, the measurement starts with an initial assessment including a 12-Channel rest and exercise ECG and questionnaire, to check the heart healthiness. At the current stage we decided to exclude participants with current or former diseases or surgeries. The actual shape of a standard BCG signal is still unknown and we want to avoid that such pre-existing conditions might affect the measurement itself or the signal outcome.

For study measurement (M1-4), four different setups are used, including two different sensor positions. For M1 and M2, the sensors are placed at the SCG positions on the sternum, the apex of the heart and the spine (see Figure 44 positions A-C). For M3 and M4, two BCG positions are selected on larger vessels on the temple and wrist with an additional BCG position on the sternum (see Figure 44 positions B, D, E). This should allow comprehensive PTT investigations.

Both odd numbered measurements are resting state recordings, while the even ones consider BCG under physical stress.

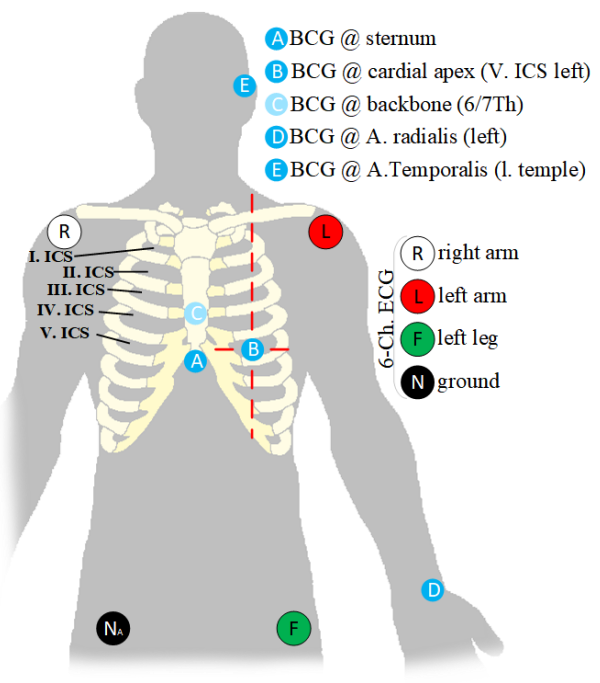

Figure 4: Positioning of the several sensors

M2 is recorded using an ergometer (2min at $40-60 \mathrm{~W}$, $1 \mathrm{~min}$ at $70-100 \mathrm{~W}, 30 \mathrm{sec}$ at $>=110 \mathrm{~W})$. M4 is measured using a treadmill $(2 \mathrm{~min}$ at $5 \mathrm{~km} / \mathrm{h}, 1 \mathrm{~min}$ at $10 \mathrm{~km} / \mathrm{h}, 30 \mathrm{sec} 15 \mathrm{~km} / \mathrm{h})$.

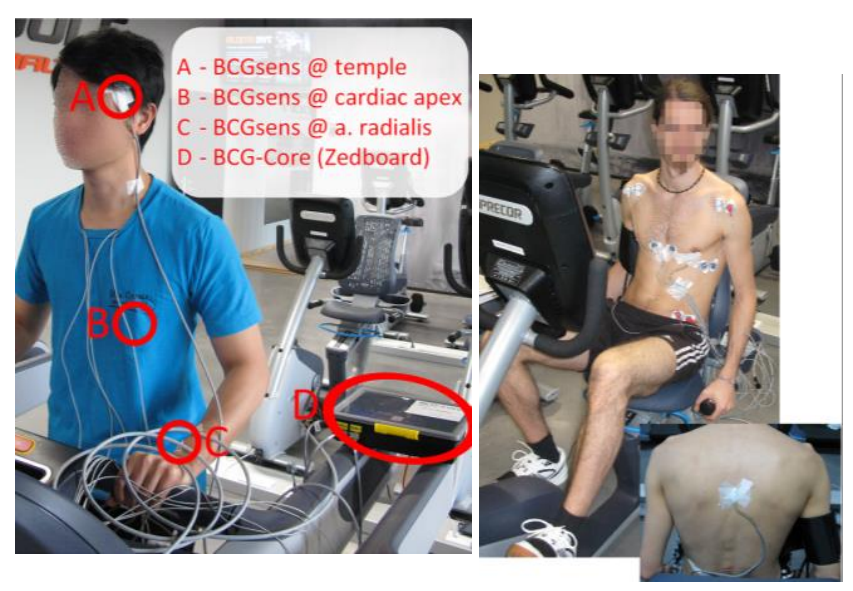

Figure 5: Subject without shirt; visible sensor position on the chest for $\mathrm{M} 1+2$ (right) and treadmill exercise, with sensor position on wrist, temple and cardiac apex (M3+4) (left)

Figure 55 (right) shows the setup for measurements M1 and $\mathrm{M} 2$ with attached sensors at different points on the chest, as well as the reference ECG. As can be seen these measurements are done in a sitting position, this can provide a steadier chest, even while exercising. On the left one subject performing M4 can be seen. The BCG acceleration sensors at subject's temple, wrist and chest, as well as the 6-channel. ECG are depicted. In the background of the picture the ergometer for the other exercise measurement can be seen.

In order to investigate the potential changes of the BCG signal over time, a number of test subjects are invited several times to perform repetitions of all four measurements.

\section{First Results}

The presented study is ongoing and this section provides first results. To improve the signal quality, we chose an internal sample frequency of $800 \mathrm{~Hz}$ for the selected sensor (KX122). Furthermore, each sensor performs an internal averaging of 128 samples, without data lost.

Due to the significantly higher working frequency of the Zedboards $(20 \mathrm{kHz})$ an oversampling factor of 11 is given for each accelerometer axis. Under the used setting, we can thus get 17,400 samples, under the conditions and limitations de- 
analog ECG channels are achieved. This leads to a data set of 27,540,000 samples for the accelerometer plus 6,912,000 samples for the reference ECG for the 3-minute measurement at rest (M1/3). In total, 1.35 - 1.55GB of data are generated per measurement and up to $6 \mathrm{~GB}$ measurement of one subject (M1- M4).

In Figure 6 a plot of raw values of one subject is depicted. For this first evaluation, we chose the resting measurements (M1) and as expected only the z-axes of the three accelerometers contain the heat related acceleration. Significant periodically recurring peaks strongly related to the reference data of the ECG (blue line) can be seen. With a slight delay of about $120 \mathrm{~ms}$, a clear BCG pattern [12] is mentionable in all $\mathrm{z}$ axes in the plot.

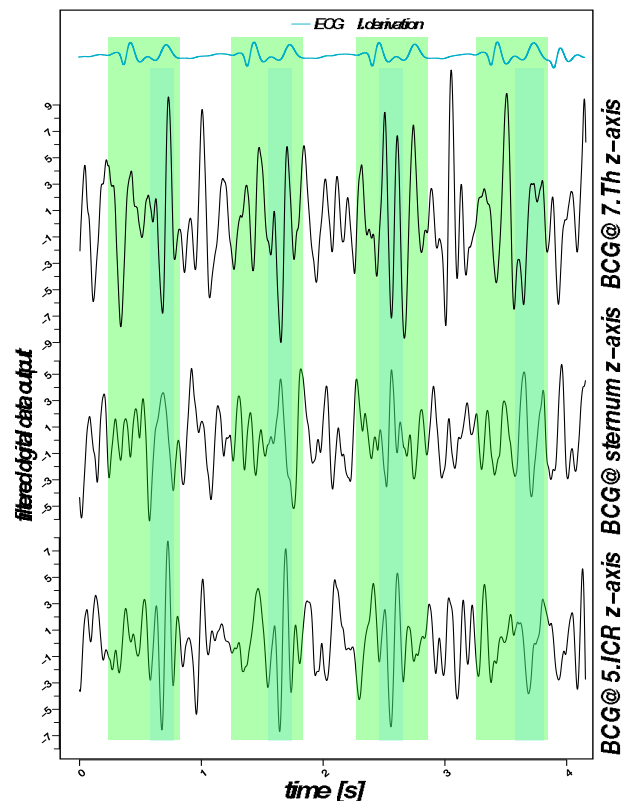

Figure 6: First simple analysis plot of the BCG signals including the reference ECG

\section{Conclusions}

In this paper we presented the motivation and the performance of our ongoing study to gather BCG ground-truth data for further examinations. With these new high-precision, synchronized and sensitive multi-position data, we want to create a subset of reference data for healthy subjects in order to provide a comparison instance for BCG research. This data may be used to provide alternative diagnostic methods, such as optimal sleep blood pressure monitoring, long term post-surgery monitoring or every-day prevention heart and health monitoring.

We presented our test setup including a custom hardware system that is able to collect BCG data with a high data quality in terms of resolution and sample rate. Simultaneously we measure an ECG reference signal.

A well-planned study considering an assessment to gather related biometric data like, e.g. habits or body shape, is currently performed. Besides measuring BCG signals at different positions of the body, also BCG under physical stress is considered.

In this study we only consider COTS acceleration sensors so that the general advantage of the selected sensors is cost efficiency, as well as availability. Hence, in the future a potential very broad use in everyday life is conceivable.

However, a deep analysis of the presented ground truth data is still to explore the full potential of the recorded data. In addition, the collected dataset is also beneficial for our current project where we are working on the use of BCG sensor technology to monitor the health condition of air passengers [11].

We collected more than 10 hours of BCG data from different subjects and positions as well as from the same subject und equal condition at different times. It is planned to provide access to this database for interested researchers in this area. Furthermore, the data diversity should be increased, for example, by recording data from children and the elderly, as well as the supplementation of diagnosed heart disease patients.

\section{Acknowledgement}

This research was supported by the German Academic Exchange Service (DAAD) and the European Institute of Innovation and Technology (EIT).

\section{References}

1. Giovangrandi L, et al.. Ballistocardiography--a method worth revisiting. Conf Proc IEEE Eng Med Biol Soc. 2011;2011:4279-82. doi:10.1109/IEMBS.2011.6091062.

2. Zanetti JM, Tavakolian K. Seismocardiography: past, present and future. Conf Proc IEEE Eng Med Biol Soc. 2013;2013:7004-7. doi:10.1109/EMBC.2013.6611170.

3. Kim C, et al.. Ballistocardiogram as Proximal Timing Reference for Pulse Transit Time Measurement: Potential for Cuffless Blood Pressure Monitoring. IEEE Trans Biomed Eng. 2015;62:2657-64. doi:10.1109/TBME.2015.2440291.

4. Di Rienzo M, et al.. 24h seismocardiogram monitoring in ambulant subjects. Conf Proc IEEE Eng Med Biol Soc. 2012;2012:5050-3. doi:10.1109/EMBC.2012.6347128.

5. Di Rienzo M, et al. Wearable seismocardiography: towards a beat-by-beat assessment of cardiac mechanics in ambulant subjects. Auton Neurosci. 2013;178:50-9. doi:10.1016/j.autneu.2013.04.005.

6. Di Rienzo M, et al. Wearable seismocardiography for the beat-to-beat assessment of cardiac intervals during sleep. Conf Proc IEEE Eng Med Biol Soc. 2014;2014:6089-91. doi:10.1109/EMBC.2014.6945018.

7. Di Rienzo M, Vaini E, Lombardi P. Use of seismocardiogram for the beat-to-beat assessment of the Pulse Transit Time: A pilot study. Conf Proc IEEE Eng Med Biol Soc. 2015;2015:7184-7. doi:10.1109/EMBC.2015.7320049.

8. Javaid AQ, Ashouri H, Inan OT. Estimating systolic time intervals during walking using wearable ballistocardiography. In: 2016 IEEE-EMBS International Conference on Biomedical and Health Informatics (BHI); Las Vegas, NV, USA. Piscataway, NJ: IEEE; 2016. p. 549-552.

doi:10.1109/BHI.2016.7455956.

9. Jähne-Raden N, et al.. BCG-mapping of the thorax using different sensors: First experiences and signal quality. In: ; Orlando, FL, USA. p. 1-3. doi:10.1109/ICSENS.2016.7808747.

10. Jähne-Raden N, Wolf K, Marschollek M. Signal Detection Accuracy of Digital Accelerometers for Ballistocardiographic Propose. In: ; 24-27 September, 2017: Computing in Cardiology; 2017. doi:10.22489/CinC.2017.027-381.

11. Jähne-Raden N, et al.. Poster: SCARAB ${ }^{2}$ - Scalable, Robust and Adaptive on Board Ballistocardiography. In: ; New York, NY, USA. p. 111-112. doi:10.1109/DCOSS.2018.00022.

12. Jähne-Raden N, Marschollek M, Kulau U, Wolf L. HeartBeat the Odds. In: Eskicioglu R, editor; Delft, Netherlands. p. 1-2. doi:10.1145/3131672.3136973.

\section{Address for correspondence}

Nico Jähne-Raden, Hannover Medical School, Carl-Neuberg-Str. 1, D-30625 Hanover, Germany, Nico.Jaehne-Raden@plri.de 